\title{
A novel constrained reconstruction model towards better information fusion in high-resolution subtomogram averaging
}

\author{
Renmin Han ${ }^{1, \dagger}$, Lun Li $\mathrm{Li}^{2,3, \dagger}$, Peng Yang ${ }^{1}$, Fa Zhang ${ }^{2, *}$, Xin Gao ${ }^{1, *}$ \\ ${ }^{1}$ King Abdullah University of Science and Technology (KAUST), Computational Bioscience Research Center (CBRC), CEMSE \\ Division, Thuwal, 23955-6900, Saudi Arabia. ${ }^{2}$ High Performance Computer Research Center, Chinese Academy of Sciences, Beijing \\ 100190, China. ${ }^{3}$ University of Chinese Academy of Sciences, Beijing, China.
}

\begin{abstract}
Motivation: Electron tomography (ET) offers a unique capacity to image biological structures in situ. However, the resolution of ET reconstructed tomograms is not comparable to that of the single-particle cryo-EM. If many copies of the object of interest are present in the tomograms, their structures can be reconstructed in the tomogram, picked, aligned and averaged to increase the signal-to-noise ratio and improve the resolution, which is known as the subtomogram averaging (STA). To date, the resolution improvement of the subtomogram averaging is still limited because each reconstructed subtomogram is of low reconstruction quality due to the missing wedge issue.

Results: In this paper, we propose a novel computational model, the constrained reconstruction model (CRM), to better recover the information from the multiple subtomograms and compensate for the missing wedge issue in each of them. CRM is supposed to produce a refined reconstruction in the final turn of subtomogram averaging after alignment, instead of the averaging. We first formulate the averaging method and our CRM as linear systems, and prove that the solution space of CRM is no larger, and in practice much smaller, than that of the averaging method. We then propose a sparse Kaczmarz algorithm to solve the formulated CRM, and further extend the solution to the simultaneous algebraic reconstruction technique (SART). Experimental results demonstrate that $\mathrm{CRM}$ can significantly alleviate the missing wedge issue and improve the final reconstruction quality. In addition, our model is robust to the number of images in each tilt series, the tilt range, and the noise level.
\end{abstract}

Availability: The Matlab code is available at https://github.com/ icthrm/constrained-reconstruction and the $\mathrm{C} / \mathrm{C}++$ codes of $\mathrm{CRM}$ SIRT and CRM-SART is available upon acceptance.

\section{INTRODUCTION}

Electron tomography (ET) is a unique technology to image biological structures, such as an entire cell, in their native environment (Frank, 2006) In ET, the object of interest is tilted for different degrees of rotations and a series of images is taken, which is then used to reconstruct the 3D structure of the object through reconstruction methods, such as backprojection algorithms based on inverse Radon transform. In the ideal case, if the tilt angles cover the full range of projections, a perfect reconstruction can be obtained. However, in practice, the tilt range of the projections is almost always limited within $\pm 40^{\circ}$ to $\pm 70^{\circ}$, which causes the missing wedge issue and leads to remarkable artifacts. Compared with other technologies, such in high-resolution imaging.

The limited range projection issue is not only a key problem in ET, but also widely exists in various fields, such as computed tomography, radial physics, ultrasonic and biomedical imaging (Shimizu et al., 1998; Wu et al., 2003; Zhu et al., 2005; Harris et al., 2017). A large number of studies have been carried out to suppress the effect of missing wedge (Carazo, 1992; Leary et al., 2013; Kovacik et al., 2014; Chen and Forster, 2014; Kupsch et al., 2016; Deng et al., 2016; Han et al., 2017). Almost all of these works attempt to introduce additional constraints representing priori knowledge about the specimen to narrow down the solution space. However, since once

${ }^{\dagger}$ These authors should be considered as joint first author

*All correspondence should be addressed to Fa Zhang (zhangfa@ict.ac.cn) and Xin Gao (xin.gao@kaust.edu.sa). as single-particle cryo-EM, this has severely limited the application of ET

the information is lost, it will never be recovered, the priori knowledge is more useful on reducing artifacts than recovering lost information.

If many copies of the object of interest are present in the tomograms, their structures can be reconstructed separately, averaged together to increase the signal-to-noise ratio and improve the resolution, which is known as the subtomogram averaging. In subtomogram averaging, the entire tomogram is firstly reconstructed from a series of projections, and then the ultra-structures (sub-volumes) containing the object of interest are picked from the reconstructed tomogram, aligned and averaged to obtain the final subtomogram. This strategy has been shown to effectively increase the signal-to-noise ratio and improve the resolution of the subtomograms. However, the reconstruction of each subtomogram still suffers from the missing wedge issue. Additionally, since the object of interest in ET is imaged in the highly noisy native environment, the surrounding background consists of not only noise but also various artifacts and other objects, which poses further challenges to subtomogram averaging.

subtomogram averaging is a quite new technology with enormous potential (Briggs, 2013). To date, several attempts have been made to increase the reconstruction quality of the subtomograms. The geometry calibration has been extensively studied to provide more accurate averaging operation (Bartesaghi et al., 2012; Xu and Alber, 2012; Xu et al., 2012; Bharat and Scheres, 2016). Other works try to change the information interpretation in tomographic reconstruction and improve the consequent subtomogram averaging (Chen and Forster, 2014; Kunz and Frangakis, 2014; Deng et al., 2016). The effect of the missing wedge has also been considered in the process of alignment and averaging, by applying an anglelimited mask to the subtomogram to compensate the "missing wedge bias" (Förster et al., 2005; Schmid and Booth, 2008; Galaz-Montoya and Ludtke, 2017). Despite the contribution of these works, the core idea remains the same, i.e., the final structure is obtained by averaging the structures of all the subtomograms.

To compose the image information of these subtomograms into a consistent structure is a typical information fusion task. In this paper, we propose a novel constrained reconstruction model (CRM) to better recover the information from the multiple subtomograms to compensate for the missing wedge issue in each of them. CRM is supposed to produce a refined reconstruction in the final turn of subtomogram averaging after alignment, instead of the traditional averaging. Here, we first formulate the averaging method and our CRM as linear systems, and demonstrate the difference between the two models. We then prove that the solution space of CRM is no larger than that of the traditional method. To solve the linear system of CRM, we propose a sparse Kaczmarz algorithm to solve the formulated CRM, and further extend the solution to the simultaneous algebraic reconstruction technique (SART). Finally, the effectiveness and robustness of CRM have been well studied and proved in both 2D and 3D cases.

\section{RELATED WORK}

Reconstruction algorithms can be categorized into direct Fourier inversion (Penczek et al., 2004; Scheres, 2012), iterative algorithms (Gordon et al., 1970; Gilbert, 1972; Andersen and Kak, 1984) and back-projection methods (De Rosier and Klug, 1968). Here, we give a brief introduction to the algebraic reconstruction technique (ART) (Gordon et al., 1970), which is a class of iterative algorithms that have been widely used in computed tomography (Andersen, 1989; Prakash et al., 2010; Beister et al., 2012).

ART is a special case of the Kaczmarz algorithm (Kaczmarz, 1937), which is an iterative algorithm for solving linear equation systems (in this work, the bold font is used to indicate vectors or matrices. A vector refers (C) The Author 2018. Published by Oxford University Press. All rights reserved. For permissions, please e-mail: journals.permissions@oup.com 
to a column vector). In ART, the values of the pixels to be reconstructed are considered as variables collected in the vector $\mathrm{x}$, the image process (i.e., projection coefficients) is described by the matrix $\boldsymbol{A}$, and the measured angular projections are collected in vector $\boldsymbol{b}$. Consequently, the tomography reconstruction problem is formulated as a system of linear equations $\boldsymbol{A x}=$ $\boldsymbol{b}$. Let $m$ be the number of rows of $\boldsymbol{A}, \boldsymbol{A}_{i}$ be the $i$ th row of the valued matrix $\boldsymbol{A}$, and $\boldsymbol{x}^{0}$ be an arbitrary initial approximation to the solution of $\boldsymbol{A x}=\boldsymbol{b}$. For $k=0,1, \ldots$, ART updates the value of $\boldsymbol{x}$ as follows:

$$
\boldsymbol{x}^{k+1}=\boldsymbol{x}^{k}+\lambda_{k} \frac{\boldsymbol{b}_{i}-\left\langle\boldsymbol{A}_{i}, \boldsymbol{x}^{k}\right\rangle}{\left\|\boldsymbol{A}_{i}\right\|^{2}} \boldsymbol{A}_{i},
$$

where $i=k \bmod m+1, b_{i}$ is the $i$ th component of vector $\boldsymbol{b}, \lambda_{k}$ is a relaxation parameter. If the linear system is consistent, $\boldsymbol{x}^{k}$ converges to the minimumnorm solution, providing that the iterations start with the zero vector.

Although the Kaczmarz's algorithm has demonstrated success in ET reconstruction, it suffers from issues of numeric stability and computational efficiency. To achieve better estimation and computational efficiency, various methods have been proposed, such as the simultaneous iterative reconstruction technique (SIRT) (Gilbert, 1972) and the simultaneous algebraic reconstruction technique (SART) (Andersen and Kak, 1984). SART is one of the most commonly used reconstruction methods. In contrast to the Kaczmarz's algorithm, SART only updates the solution once per copy, after solving all the projections of a copy.

\section{METHODS}

Since this work focuses on the information fusion (reconstruction) in subtomogram averaging, we assume that the projections have been posed in the correct geometry. Without loss of generality and for the simplicity of presentation, we discuss the $2 \mathrm{D}$ condition first and then straightforward to generalize techniques to the $3 \mathrm{D}$ case.

\subsection{Constrained reconstruction model}

We first prove that the solution space of CRM is no larger, and in practice much smaller, than that of the traditional method.

3.1.1 Problem description For a compactly supported continuous function $h: \mathbb{R}^{2} \rightarrow \mathbb{R}, N$ copies of $h$ exist and each copy $h_{i}$ has surrounding (background) $g_{i}$. For each copy, the copy $h_{i}$ and background $g_{i}$ can be clearly separated in the space $\mathbb{R}^{2}$, i.e.,

$$
\begin{array}{r}
h_{i}, g_{i}: \mathbb{R}^{2} \rightarrow \mathbb{R} ; i=1, \ldots, N, \\
\operatorname{supp}\left(h_{i}\right) \cap \operatorname{supp}\left(g_{i}\right)=\varnothing ; i=1, \ldots, N,
\end{array}
$$

where $\operatorname{supp}()$ denotes the set-theoretic support. Here, the background denotes the information in the tomogram surrounding the particle. We should try to estimate the background so we can subtract these information from the structure, to avoid artifects.

Given the stained function $\left\{f_{i} \stackrel{\text { def }}{=} h_{i}+g_{i}\right\}$, each $f_{i}$ is tilted for a limited range of angles and projected. The corresponding measured projections for the $i$ th copy are

$$
p_{i}\left(\theta_{i j}, s\right)=R f_{i}\left(\theta_{i j}, s\right) ; i=1, \ldots, N, \theta_{i j} \in \Theta_{i}, j \leq m,
$$

where $R f$ presents the Radon transform of function $f, \theta_{i j}$ is the $j$ th projection angle for $f_{i}, \Theta_{i}$ is the limited tilt angle range, and $m$ is the number of projections in each copy (if the numbers of projections of different copies are different, $m$ is the maximum number among all the copies). The goal is to estimate $h$ from the $N$ sets of measured projections $\left\{p_{i}\left(\theta_{i j}, s\right)\right\}$.

For $h$, the conventional solution is to first solve the inverse Radon transform $R_{i n v}\left(p_{i}\right)$ for each stained function $f_{i}$ to obtain $\hat{h}_{i}$ (the estimation of $h_{i}$ ), and then average all the $\hat{h}_{i}$ to obtain $\hat{h}$ (the estimation of $h$ ). That is,

$$
\hat{h}=\frac{1}{N} \sum_{i=1}^{N} \hat{h}_{i}=\frac{1}{N} \sum_{i=1}^{N}\left(1_{\operatorname{supp}(f)} \otimes R_{\text {inv }}\left(p_{i}\right)\right),
$$

where $\otimes$ presents the Hadamard product.

3.1.2 Constrained reconstruction as linear systems In the discrete condition, Radon transform can be reduced to a linear system. Fig. 1 illustrates the main difference between the traditional subtomogram averaging method and the proposed CRM. In the traditional method, each subtomogram is reconstructed separately and independently, and all of them are averaged together to obtain the final reconstruction. However, the

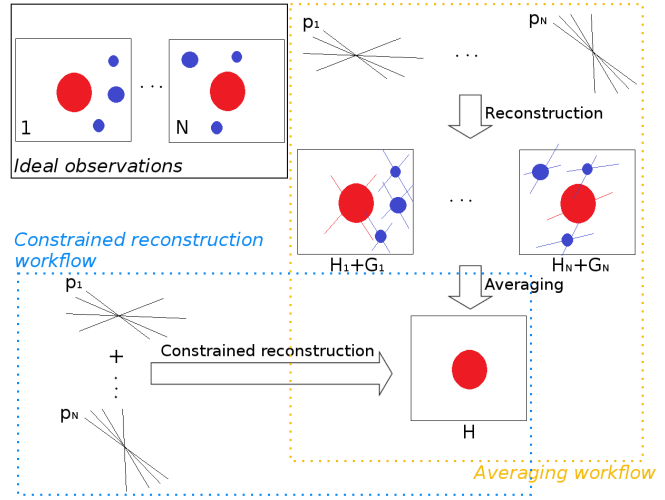

Fig. 1: Illustration of the difference between the averaging-based method and the proposed constrained reconstruction model (CRM).

missing wedge issue still exists in each reconstruction and is thus inherited to the final estimation. In contrast, CRM constrains all the subtomograms together, by which it can effectively combine the information from different copies to compensate for the missing wedge issue. We give the formulation of both the traditional method and CRM in linear systems as follows:

For the $i$ th copy, we collect the measured angular projections $p_{i}\left(\theta_{i j}, s\right), j=1, \ldots, m$ in a vector $\boldsymbol{b}_{i}$. Define vector $\boldsymbol{f}_{i}=\left[\boldsymbol{h}_{i}^{T}, \boldsymbol{g}_{i}^{T}\right]^{T}$ and matrix $\boldsymbol{A}_{i}=\left[\boldsymbol{D}_{i}, \boldsymbol{B}_{i}\right]$, where vector $\boldsymbol{h}_{i}$ represents the pixels covered by function $h_{i}$, vector $\boldsymbol{g}_{i}$ represents the pixels covered by function $g_{i}$, matrix $\boldsymbol{D}_{i}$ represents the projection coefficients operated on $\boldsymbol{h}_{i}$, and matrix $\boldsymbol{B}_{i}$ represents the projection coefficients operated on $\boldsymbol{g}_{i}$. The reconstruction problem for the $i$ th copy is to obtain $\boldsymbol{f}_{i}$ by solving $\boldsymbol{A}_{i} \boldsymbol{f}_{i}=\boldsymbol{b}_{i}$.

The averaging method can be formulated as the following linear system:

$$
\left\{\begin{array}{c}
\boldsymbol{D}_{1} \boldsymbol{h}_{1}+\boldsymbol{B}_{1} \boldsymbol{g}_{1}=\boldsymbol{b}_{1} \\
\vdots \\
\boldsymbol{D}_{N} \boldsymbol{h}_{N}+\boldsymbol{B}_{N} \boldsymbol{g}_{N}=\boldsymbol{b}_{N} \\
\boldsymbol{h}=\frac{1}{N} \sum_{i=1}^{N} \boldsymbol{h}_{i}
\end{array}\right.
$$

where vector $\boldsymbol{h}$ is the final discrete estimation for function $h$.

The constrained reconstruction model introduces the prior that $h_{1}=$ $h_{2} \ldots=h_{N}=h$ and can be formulated as:

$$
\left\{\begin{array}{c}
\boldsymbol{D}_{1} \boldsymbol{h}+\boldsymbol{B}_{1} \boldsymbol{g}_{1}=\boldsymbol{b}_{1} \\
\vdots \\
\boldsymbol{D}_{N} \boldsymbol{h}+\boldsymbol{B}_{N} \boldsymbol{g}_{N}=\boldsymbol{b}_{N}
\end{array}\right.
$$

Here, the estimation $\left\{\boldsymbol{h}_{i}\right\}$ is directly replaced by $\boldsymbol{h}$. Therefore, the reconstruction is based on all the projections simultaneously. We give the following theorem to guarantee the feasibility of efficiently solving the linear system in (6).

THEOREM 1. The solution space of the constrained reconstruction model in (6) is no larger than that of the averaging method in (5).

Proof. By extending the linear systems in (5) and (6), we can rewrite the formulation for the averaging method as:

$$
\left[\begin{array}{ccccccccc}
\boldsymbol{D}_{1} & \boldsymbol{B}_{1} & \cdots & 0 & 0 & \cdots & 0 & 0 & 0 \\
\vdots & \vdots & \ddots & \vdots & \vdots & & \vdots & \vdots & \vdots \\
0 & 0 & \cdots & \boldsymbol{D}_{i} & \boldsymbol{B}_{i} & \cdots & 0 & 0 & 0 \\
\vdots & \vdots & & \vdots & \vdots & \ddots & \vdots & \vdots & \vdots \\
0 & 0 & \cdots & 0 & 0 & \cdots & \boldsymbol{D}_{N} & \boldsymbol{B}_{N} & 0 \\
\boldsymbol{I} & 0 & \cdots & \boldsymbol{I} & 0 & \cdots & \boldsymbol{I} & 0 & -N \boldsymbol{I}
\end{array}\right]\left[\begin{array}{c}
\boldsymbol{h}_{1} \\
\boldsymbol{g}_{1} \\
\vdots \\
\boldsymbol{h}_{i} \\
\boldsymbol{g}_{i} \\
\vdots \\
\boldsymbol{h}_{N} \\
\boldsymbol{g}_{N} \\
\boldsymbol{h}
\end{array}\right]=\left[\begin{array}{c}
\boldsymbol{b}_{1} \\
\vdots \\
\boldsymbol{b}_{i} \\
\vdots \\
\boldsymbol{b}_{N} \\
0
\end{array}\right]
$$

and rewrite the formulation for CRM as:

$$
\left[\begin{array}{cccccc}
\boldsymbol{D}_{1} & \boldsymbol{B}_{1} & \cdots & 0 & \cdots & 0 \\
\vdots & \ddots & \vdots & & \vdots & \vdots \\
\boldsymbol{D}_{i} & 0 & \cdots & \boldsymbol{B}_{i} & \cdots & 0 \\
\vdots & & \vdots & \ddots & \vdots & \vdots \\
\boldsymbol{D}_{N} & 0 & \cdots & 0 & \cdots & \boldsymbol{B}_{N}
\end{array}\right]\left[\begin{array}{c}
\boldsymbol{h} \\
\boldsymbol{g}_{1} \\
\vdots \\
\boldsymbol{g}_{i} \\
\vdots \\
\boldsymbol{g}_{N}
\end{array}\right]=\left[\begin{array}{c}
\boldsymbol{b}_{1} \\
\vdots \\
\boldsymbol{b}_{i} \\
\vdots \\
\boldsymbol{b}_{N}
\end{array}\right] .
$$

Here, we denote the left parameter matrix of Eq. (7) as $\boldsymbol{A}_{\text {avg }}$ and the parameter matrix of Eq. (8) as $\boldsymbol{A}_{C R M}$. For an underdetermined $m \times n$ linear 
system $\boldsymbol{A} \boldsymbol{x}=\boldsymbol{b}$, the degree of freedom depends on the number of free variables or the span of the left-hand matrix, i.e., $\operatorname{dim}(\boldsymbol{A})=n-\operatorname{rank}(\boldsymbol{A})$, where $\operatorname{dim}(\boldsymbol{A})$ represents the dimension of the solution space. Denoting the attribute number in $\boldsymbol{g}_{i}, \boldsymbol{h}_{i}$ and $\boldsymbol{h}$ as $n_{g_{i}}, n_{h_{i}}$ and $n_{h}$ respectively, where $n_{h}=n_{h_{i}}, i=1, \ldots, N$, we get the dimension of the solution space for the averaging method and CRM as

$$
\operatorname{dim}\left(\boldsymbol{A}_{a v g}\right)=n_{h}+\sum_{i=1}^{N}\left(n_{g_{i}}+n_{h_{i}}\right)-\operatorname{rank}\left(\boldsymbol{A}_{a v g}\right),
$$

and

$$
\operatorname{dim}\left(\boldsymbol{A}_{C R M}\right)=n_{h}+\sum_{i=1}^{N} n_{g_{i}}-\operatorname{rank}\left(\boldsymbol{A}_{C R M}\right) .
$$

Considering $\operatorname{rank}\left(\boldsymbol{A}_{\text {avg }}\right)=\sum_{i=1}^{N} \operatorname{rank}\left(\left[\boldsymbol{D}_{i}, \boldsymbol{B}_{i}\right]\right)+n_{h}$ and $n(h)=n\left(h_{i}\right), i=$ $1, \ldots, N$, the dimension of the solution space for the averaging method can be simplified as

$$
\operatorname{dim}\left(\boldsymbol{A}_{\text {avg }}\right)=N \cdot n_{h}+\sum_{i=1}^{N} n_{g_{i}}-\sum_{i=1}^{N} \operatorname{rank}\left(\left[\boldsymbol{D}_{i}, \boldsymbol{B}_{i}\right]\right) .
$$

Considering the fact $\operatorname{rank}\left(\left[\begin{array}{ll}\boldsymbol{P} & \boldsymbol{S} \\ 0 & \boldsymbol{Q}\end{array}\right]\right) \geq \operatorname{rank}(\boldsymbol{P})+\operatorname{rank}(\boldsymbol{Q})$, we have $\operatorname{rank}\left(\boldsymbol{A}_{C R M}\right) \geq \operatorname{rank}\left(\left[\boldsymbol{D}_{1}, \boldsymbol{B}_{1}\right]\right)+\sum_{i=2}^{N} \operatorname{rank}\left(\boldsymbol{B}_{i}\right)$. Therefore,

$\operatorname{dim}\left(\boldsymbol{A}_{C R M}\right) \leq n_{h}+\sum_{i=1}^{N} n_{g_{i}}-\operatorname{rank}\left(\left[\boldsymbol{D}_{1}, \boldsymbol{B}_{1}\right]\right)-\sum_{i=2}^{N} \operatorname{rank}\left(\boldsymbol{B}_{i}\right)$.

By subtracting Eq. (12) from Eq. (11), we have

$$
\begin{aligned}
\operatorname{dim}\left(\boldsymbol{A}_{\text {avg }}\right)-\operatorname{dim}\left(\boldsymbol{A}_{C R M}\right) & \geq \\
(N-1) \cdot n_{h} & +\sum_{i=2}^{N}\left(\operatorname{rank}\left(\boldsymbol{B}_{i}\right)-\operatorname{rank}\left(\left[\boldsymbol{D}_{i}, \boldsymbol{B}_{i}\right]\right)\right) .
\end{aligned}
$$

Rewriting the inequality and considering $n_{h}=n_{h_{i}} \geq \operatorname{rank}\left(\boldsymbol{D}_{i}\right)$, we have

$$
\begin{gathered}
\operatorname{dim}\left(\boldsymbol{A}_{\text {avg }}\right)-\operatorname{dim}\left(\boldsymbol{A}_{C R M}\right) \geq \sum_{i=2}^{N}\left(n_{h_{i}}-\operatorname{rank}\left(\boldsymbol{D}_{i}\right)\right)+ \\
\sum_{i=2}^{N}\left(\operatorname{rank}\left(\boldsymbol{D}_{i}\right)+\operatorname{rank}\left(\boldsymbol{B}_{i}\right)-\operatorname{rank}\left(\left[\boldsymbol{D}_{i}, \boldsymbol{B}_{i}\right]\right)\right) \geq 0 .
\end{gathered}
$$

By this, we have proved that the solution space of CRM is no larger than that of the averaging method.

Here, we should note that the main difference between $\operatorname{dim}\left(\boldsymbol{A}_{\text {avg }}\right)$ and $\operatorname{dim}\left(\boldsymbol{A}_{C R M}\right)$ comes from $n_{h_{i}}-\operatorname{rank}\left(\boldsymbol{D}_{i}\right)$. In real-world data sets, the range of tilt angles is limited and the sampling is discrete, which makes $n_{h_{i}} \gg$ $\operatorname{rank}\left(\boldsymbol{D}_{i}\right)$ and results in the missing wedge issue. Therefore, in practice $\operatorname{dim}\left(\boldsymbol{A}_{C R M}\right)$ should be much smaller than $\operatorname{dim}\left(\boldsymbol{A}_{\text {avg }}\right)$ and thus leads to better estimations.

\subsection{Solution to the constrained reconstruction model}

Considering an L2-norm minimized solution, the optimization of Eq. (6) (or Eq. (8)) is to find such $\boldsymbol{h}_{i}$ and $\boldsymbol{g}_{i}$ to minimize the total deviation. Therefore, the objective function can be written as

$$
\begin{gathered}
\min _{\boldsymbol{h}_{i}, \boldsymbol{g}_{i}} \sum_{i=1}^{N}\left\|\boldsymbol{D}_{i} \boldsymbol{h}_{i}+\boldsymbol{B}_{i} \boldsymbol{g}_{i}-\boldsymbol{b}_{i}\right\|_{2}^{2}, \\
\text { s.t. } \quad \boldsymbol{h}_{i}=\boldsymbol{h}_{i+1} ; i=1, \ldots, N-1 .
\end{gathered}
$$

Several methods can be applied to solve the optimization problem, such as least square estimation (LSE) and the Kaczmarz's algorithm. Because LSE needs to solve the inversion of the entire matrix in Eq. (8), it is computationally costly, especially when a large number of copies exist. On the other hand, Kaczmarz's algorithm is an iterative algorithm for solving linear equation systems. Thus it is also applicable to Eq. (8) without large changes. However, a direct implementation of the Kaczmarz's algorithm is still costly in terms of runtime and memory. Here we first propose a novel sparse Kaczmarz's algorithm to solve the problem, then extend the solution to SART and finally propose the overall framework to solve the CRM.

3.2.1 Sparse Kaczmarzs algorithm to solve CRM Considering the linear system in Eq. (8), let $\boldsymbol{A}_{C R M}=\left[\boldsymbol{A}_{1}, \boldsymbol{A}_{2}, \ldots, \boldsymbol{A}_{N}\right]^{T}, \overline{\boldsymbol{b}}=\left[\boldsymbol{b}_{1}, \boldsymbol{b}_{2}, \ldots, \boldsymbol{b}_{N}\right]^{T}$, $\boldsymbol{D}_{i}=\left[\boldsymbol{D}_{i, 1}, \ldots, \boldsymbol{D}_{i, m}\right]^{T}, \boldsymbol{B}_{i}=\left[\boldsymbol{B}_{i, 1}, \ldots, \boldsymbol{B}_{i, m}\right]^{T}$, and $\boldsymbol{b}_{i}=\left[\boldsymbol{b}_{i, 1}, \ldots, \boldsymbol{b}_{i, m}\right]^{T}$, where $\boldsymbol{A}_{i}$ is the $i$ th component of $\boldsymbol{A}_{C R M}, \boldsymbol{b}_{i}$ is the $i$ th component of $\overline{\boldsymbol{b}}, \boldsymbol{D}_{i, l}$ is the $l$ th row of $\boldsymbol{D}_{i}, \boldsymbol{B}_{i, l}$ is the $l$ th row of $\boldsymbol{B}_{i}$, and $\boldsymbol{b}_{i, l}$ is the $l$ th row of $\boldsymbol{b}_{i}$. Here, we iteratively solve the linear system with the Kaczmarz's algorithm.
Let $\boldsymbol{f}^{(k, j)}=\left[\boldsymbol{h}^{(k, j)}, \boldsymbol{g}^{(k, j)}\right]$ be the reconstruction from the $j$ th projection image from the $i$ th iteration, where $j=m p+q(p=0, \ldots, N-1, q=0, \ldots, m-$ 1). Starting from a random solution, $\boldsymbol{f}^{(k, j)}$ can be updated as following:

$$
\boldsymbol{f}^{(k, j)}=\left\{\begin{array}{l}
\boldsymbol{f}^{(k, j-1)}+\frac{\boldsymbol{b}_{j}-\boldsymbol{A}_{j} \boldsymbol{f}^{(k, j-1)}}{\left\|\boldsymbol{A}_{j}\right\|^{2}} \boldsymbol{A}_{j}^{T}, \quad j=2,3, \ldots, m N, \\
\boldsymbol{f}^{(k-1, m \cdot N)}+\frac{\boldsymbol{b}_{j}-\boldsymbol{A}_{j} \boldsymbol{f}^{(k-1, m \cdot N)}}{\left\|\boldsymbol{A}_{j}\right\|^{2}} \boldsymbol{A}_{j}^{T}, \quad j=1 .
\end{array}\right.
$$

Due to the sparsity of matrix $\boldsymbol{A}$, Eq. (16) can be further simplified. For the $j$ th row of $\boldsymbol{A}, \boldsymbol{A}_{j}$ (i.e., the $q$ th projection image in the $p$ th copy), we have $\boldsymbol{A}_{j}=\left[\boldsymbol{D}_{p+1, q}, \mathbf{0}, \boldsymbol{B}_{p+1, q}, \mathbf{0}\right]$, which is sparse. Substituting $\boldsymbol{A}_{j}$ into Eq. (16) we can obtain

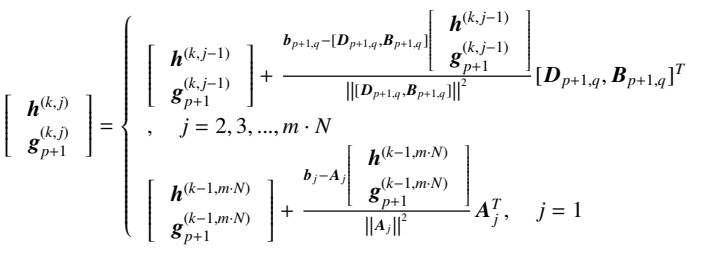

which is equivalent to solving $\boldsymbol{D}_{i} \boldsymbol{h}+\boldsymbol{B}_{i} \boldsymbol{g}_{i}=\boldsymbol{b}_{i}$ and then transporting the solved $\hat{\boldsymbol{h}}_{i}$ to $\boldsymbol{D}_{i+1} \boldsymbol{h}+\boldsymbol{B}_{i+1} \boldsymbol{g}_{i+1}=\boldsymbol{b}_{i+1}$ for $i=1,2, \ldots, N-1$, until the computation converges or the maximal number of iterations is reached.

Algorithm 1 outlines the proposed sparse Kaczmarz's algorithm to solve the constrained reconstruction model. In Algorithm $1, \boldsymbol{h}^{k}$ is the finally reconstructed subtomogram for the $k$ th iteration by Eq. (17) and $g_{i}^{k}$ is the finally calculated background for the $i$ th copy in the $k$ th iteration by Eq. (17). It should be noted that the derivation above does not introduce any additional assumptions or constraints. Therefore, sparse Kaczmarz's algorithm will give the same solution as the original Kaczmarz's algorithm.

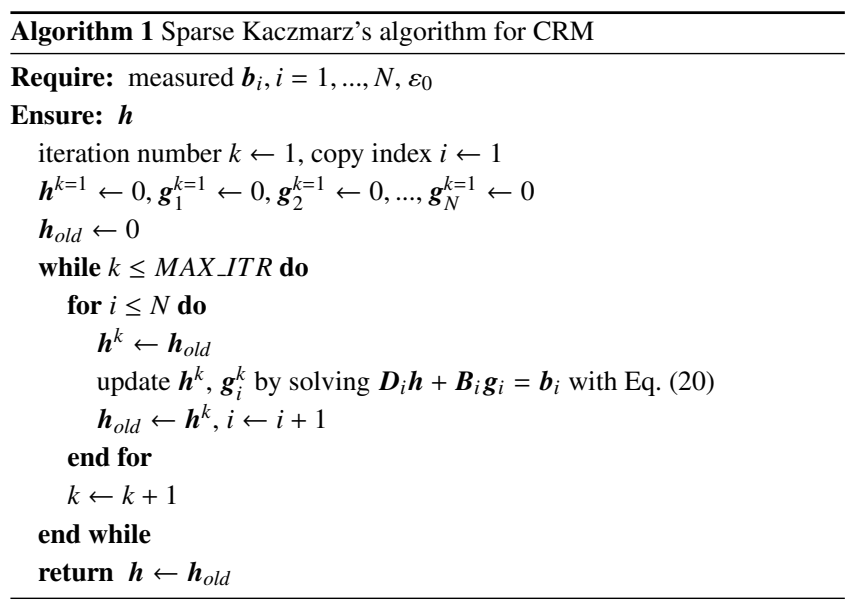

3.2.2 Sparse SART and the overall framework to solve CRM Comparing to the Kaczmarz's methods, SART is more efficient and is thus widely applied in ET reconstruction. The main difference between the Kaczmarz's algorithm and SART is that the former needs to update the solution in each projection image of each copy, whereas the latter only updates the solution once per copy after solving all the projections of a copy.

We start from the case where $N=2$, i.e., two copies of the object of interest exist, and then generalize the conclusion to any arbitrary finite $N$. The CRM formulation in the case of $N=2$ can be written as:

$$
\left[\begin{array}{ccc}
\boldsymbol{D}_{1} & \boldsymbol{B}_{1} & 0 \\
\boldsymbol{D}_{2} & 0 & \boldsymbol{B}_{2}
\end{array}\right]\left[\begin{array}{l}
\boldsymbol{h} \\
\boldsymbol{g}_{1} \\
\boldsymbol{g}_{2}
\end{array}\right]=\left[\begin{array}{l}
\boldsymbol{b}_{1} \\
\boldsymbol{b}_{2}
\end{array}\right]
$$

Let $\boldsymbol{A}=\left[\boldsymbol{D}_{1}, \boldsymbol{B}_{1}, 0 ; \boldsymbol{D}_{2}, 0, \boldsymbol{B}_{2}\right], \boldsymbol{x}=\left[\boldsymbol{h}, \boldsymbol{g}_{1}, \boldsymbol{g}_{2}\right]^{T}$ and $\boldsymbol{b}=\left[\boldsymbol{b}_{1}, \boldsymbol{b}_{2}\right]^{T}$, SART solves the linear system by the following iterative procedure:

$$
\boldsymbol{x}^{k+1}=\boldsymbol{x}^{k}+w \boldsymbol{V}^{-1} \boldsymbol{A}^{T} \boldsymbol{W}^{-1}\left(\boldsymbol{b}-\boldsymbol{A} \boldsymbol{x}^{k}\right), k=0,1,2, \ldots,
$$

where $w$ is the relaxation parameter that belongs to $(0,2)$ (Jiang and Wang, 2003), $\boldsymbol{V}$ is a diagonal matrix with $\boldsymbol{A}_{+, j}=\sum_{i} \boldsymbol{A}_{i, j}, \boldsymbol{W}$ is a diagonal matrix with $\boldsymbol{A}_{i,+}=\sum_{j} \boldsymbol{A}_{i, j}$

Let $\boldsymbol{A}_{1}=\left[\boldsymbol{D}_{1}, \boldsymbol{B}_{1}, 0\right], \boldsymbol{A}_{2}=\left[\boldsymbol{D}_{2}, 0, \boldsymbol{B}_{2}\right], \boldsymbol{V}=\operatorname{diag}\left(\boldsymbol{V}_{\boldsymbol{D}}, \boldsymbol{V}_{\boldsymbol{B}_{1}}, \boldsymbol{V}_{\boldsymbol{B}_{2}}\right)$ and $\boldsymbol{W}=\operatorname{diag}\left(\boldsymbol{W}_{1}, \boldsymbol{W}_{2}\right)$. The iterative procedure in Eq. (19) can be rewritten as: 


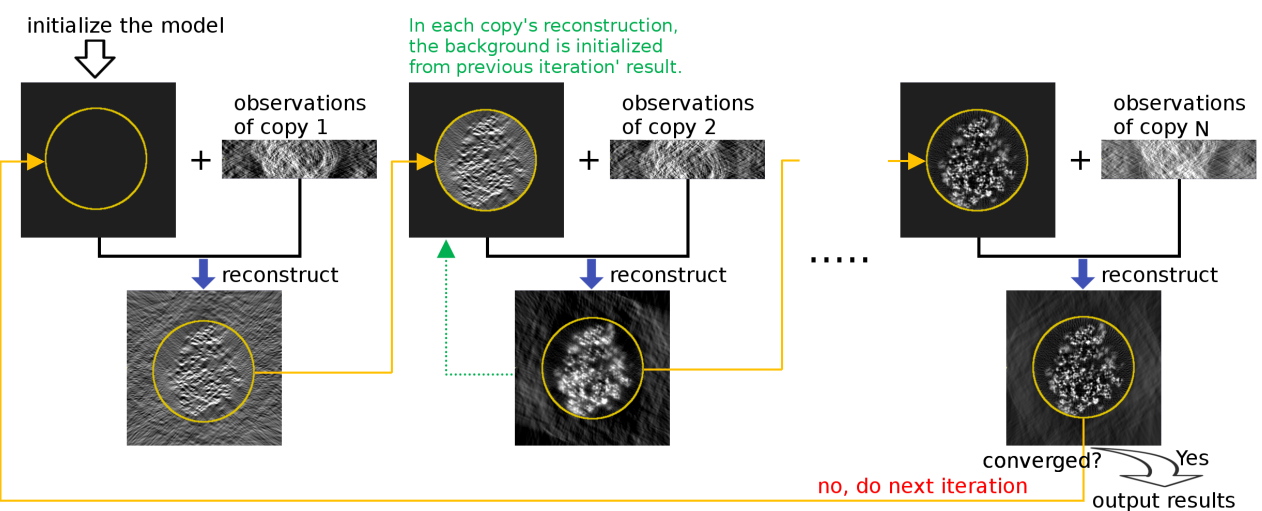

Fig. 2: Illustration of the workflow of the sparse SART to solve the constrained reconstruction model. For a copy in a certain iteration, the background is initialized by the background of the same copy from the previous iteration, and the object of interest is initialized by the object from the previous copy in the same iteration.

$$
\begin{aligned}
& \boldsymbol{x}^{k+1}= \\
& \boldsymbol{x}^{k}+w\left[\begin{array}{lll}
\boldsymbol{V}_{\boldsymbol{D}} & & \\
& \boldsymbol{V}_{\boldsymbol{B}_{1}} & \\
& & \boldsymbol{V}_{\boldsymbol{B}_{2}}
\end{array}\right]^{-1}\left[\begin{array}{l}
\boldsymbol{A}_{1} \\
\boldsymbol{A}_{2}
\end{array}\right]^{T}\left[\begin{array}{ll}
\boldsymbol{W}_{1} & \\
& \boldsymbol{W}_{2}
\end{array}\right]^{-1}\left(\left[\begin{array}{l}
\boldsymbol{b}_{1} \\
\boldsymbol{b}_{2}
\end{array}\right]-\left[\begin{array}{l}
\boldsymbol{A}_{1} \\
\boldsymbol{A}_{2}
\end{array}\right] \boldsymbol{x}^{k}\right) \\
& =\boldsymbol{x}^{k}+w\left[\begin{array}{ccc}
\boldsymbol{V}_{\boldsymbol{D}}^{-1} & & \\
& \boldsymbol{V}_{\boldsymbol{B}_{1}}^{-1} & \\
& & \boldsymbol{V}_{\boldsymbol{B}_{2}}^{-1}
\end{array}\right] \boldsymbol{A}_{1}^{T} \boldsymbol{W}_{1}^{-1}\left(\boldsymbol{b}_{1}-\boldsymbol{A}_{1} \boldsymbol{x}^{k}\right) \\
& +w\left[\begin{array}{ccc}
\boldsymbol{V}_{\boldsymbol{D}}^{-1} & & \\
& \boldsymbol{V}_{\boldsymbol{B}_{1}}^{-1} & \\
& & \boldsymbol{V}_{\boldsymbol{B}_{2}}^{-1}
\end{array}\right] \boldsymbol{A}_{2}^{T} \boldsymbol{W}_{2}^{-1}\left(\boldsymbol{b}_{2}-\boldsymbol{A}_{2} \boldsymbol{x}^{k}\right) .
\end{aligned}
$$

Here, it should be noted that

$$
\begin{aligned}
& w\left[\begin{array}{ccc}
\boldsymbol{V}_{\boldsymbol{D}}^{-1} & & \\
& \boldsymbol{V}_{\boldsymbol{B}_{1}}^{-1} & \\
& \boldsymbol{V}_{\boldsymbol{B}_{2}}^{-1}
\end{array}\right] \boldsymbol{A}_{1}^{T} \boldsymbol{W}_{1}^{-1}\left(\boldsymbol{b}_{1}-\boldsymbol{A}_{1} \boldsymbol{x}^{k}\right) \\
& =w\left[\begin{array}{ccc}
\boldsymbol{V}_{\boldsymbol{D}}^{-1} & & \\
& \boldsymbol{V}_{\boldsymbol{B}_{1}}^{-1} & \\
& & \boldsymbol{V}_{\boldsymbol{B}_{2}}^{-1}
\end{array}\right]\left[\begin{array}{c}
\boldsymbol{D}_{1}^{T} \\
\boldsymbol{B}_{1}^{T} \\
0
\end{array}\right] \boldsymbol{W}_{1}^{-1}\left(\boldsymbol{b}_{1}-\left[\begin{array}{lll}
\boldsymbol{D}_{1} & \boldsymbol{B}_{1} & 0
\end{array}\right]\left[\begin{array}{c}
\boldsymbol{h}^{k} \\
\boldsymbol{g}_{1}^{k} \\
\boldsymbol{g}_{2}^{k}
\end{array}\right]\right) \\
& =w\left[\begin{array}{ccc}
\boldsymbol{V}_{\boldsymbol{D}}^{-1} & & \\
& \boldsymbol{V}_{\boldsymbol{B}_{1}}^{-1} & \\
& & 0
\end{array}\right]\left[\begin{array}{c}
\boldsymbol{D}_{1}^{T} \\
\boldsymbol{B}_{1}^{T} \\
0
\end{array}\right] \boldsymbol{W}_{1}^{-1}\left(\boldsymbol{b}_{1}-\left[\begin{array}{lll}
\boldsymbol{D}_{1} & \boldsymbol{B}_{1} & 0
\end{array}\right]\left[\begin{array}{c}
\boldsymbol{h}^{k} \\
\boldsymbol{g}_{1}^{k} \\
0
\end{array}\right]\right)
\end{aligned}
$$

3. Solve the $i$ th reconstruction with the iterative procedure described in Eq. (19), and $i=i+1$;

4.Repeat Step 2 and Step 3 until all the copies have been updated;

5.Go to next iteration $(k=k+1)$ until convergence or the maximal number of iterations is reached.

\section{EXPERIMENTAL RESULTS}

We first prove the effectiveness of our method on two data sets: the widely-used Shepp-Logan head phantom (Shepp and Logan, 1974) and the ribosome structure downloaded from EMDB-3489 (the center slice is used) (James et al., 2016) (Fig. 3). The sizes of the two images are $320 \times 320$ and $200 \times 200$ pixels, respectively. The input data sets of projections were simulated as follows. We got a number of copies of the seed image. Each image was stained by various types of surroundings around the object of interest, including the black blocks and noise. Since for each stained image, we could only obtain a limited range of projections, we generated a series of limited tilt angles and projected the image according to these tilt angles, resulting in a series of Radon transformed images.
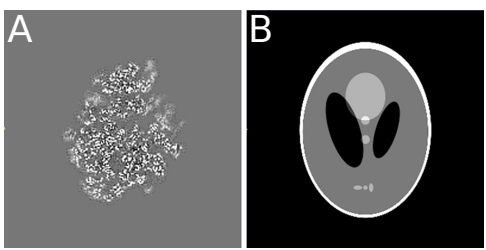

$$
\begin{aligned}
& w\left[\begin{array}{ccc}
\boldsymbol{V}_{\boldsymbol{D}}^{-1} & & \\
& \boldsymbol{V}_{\boldsymbol{B}_{1}}^{-1} & \\
& \boldsymbol{V}_{\boldsymbol{B}_{2}}^{-1}
\end{array}\right] \boldsymbol{A}_{2}^{T} \boldsymbol{W}_{2}^{-1}\left(\boldsymbol{b}_{2}-\boldsymbol{A}_{2} \boldsymbol{x}^{k}\right) \\
& =w\left[\begin{array}{ccc}
\boldsymbol{V}_{\boldsymbol{D}}^{-1} & & \\
& 0 & \\
& & \boldsymbol{V}_{\boldsymbol{B}_{2}}^{-1}
\end{array}\right]\left[\begin{array}{c}
\boldsymbol{D}_{2}^{T} \\
0 \\
\boldsymbol{B}_{2}^{T}
\end{array}\right] \boldsymbol{W}_{2}^{-1}\left(\boldsymbol{b}_{2}-\left[\begin{array}{lll}
\boldsymbol{D}_{2} & 0 & \boldsymbol{B}_{2}
\end{array}\right]\left[\begin{array}{c}
\boldsymbol{h}^{k} \\
0 \\
\boldsymbol{g}_{2}^{k}
\end{array}\right]\right) .
\end{aligned}
$$
slice of the ribosome structure (EMDB 3489 ).

The results demonstrated below are based on the sparse SART solution to CRM. The results generated by the simple averaging method and the averaging in Fourier space with a mask considered the missing wedge (masked averaging in Fourier) (Schmid and Booth, 2008) are also demonstrated as a comparison. For the simple averaging method, the copies will be added and averaged in the real space; for the method of masked averaging in Fourier, the copies will be transformed into Fourier space, be weighted with a mask according to the missing wedge, averaged in Fourier space with the synthesized weight and then transformed back into real space. Furthermore, our framework can be easily migrated to other reconstruction techniques (e.g., SIRT version in Supplementary Materials).

\subsection{Visual evaluation}

To visually evaluate the performance of our method, we set $N=3$ for the first experiment, which means we have three subtomograms to be averaged. We stained the Shepp-Logan phantom with blocks and stained the ribosome structure with random noise. We limited the tilt angle range to $\pm 50^{\circ}$ with an interval of $3^{\circ}$ for the experiment in Figure 4, 5 and 6. We compared our constrained reconstruction model with the simple averaging method and the masked averaging in Fourier. Our CRM was solved by the sparse SART (Section 3.2.2) with 20 iterations and the relaxation parameter to be 0.2 . For the sake of fair comparison, the reconstruction of each subtomogram in the simple averaging method and the masked averaging in Fourier was also done by SART with the same parameters. 


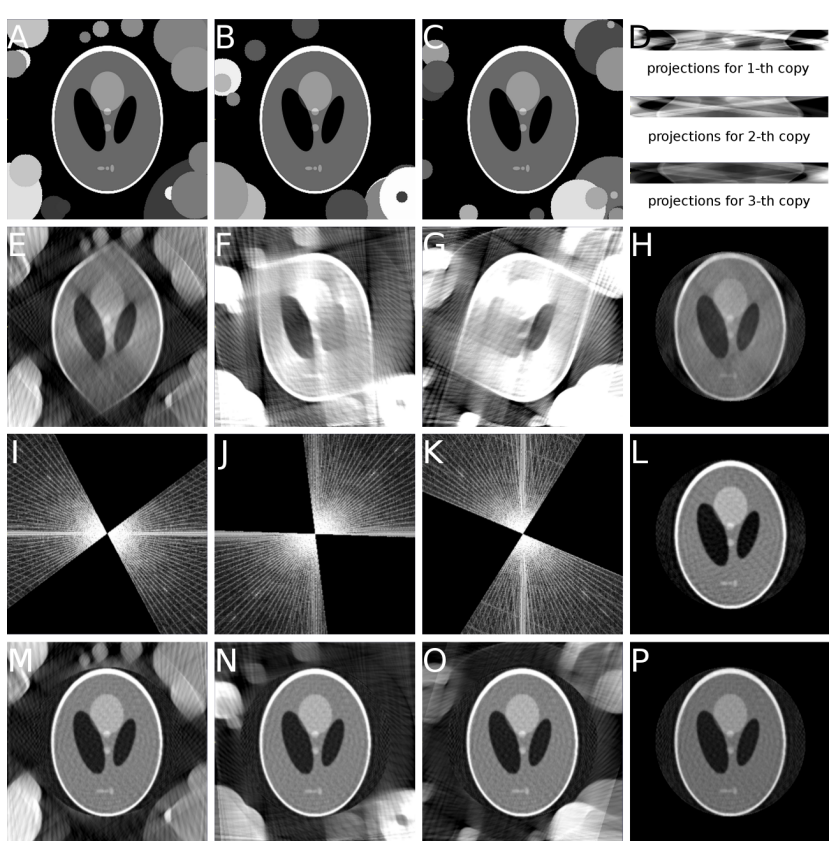

Fig. 4: Comparison of the reconstruction performance on the Shepp-Logan phantom data set. The first row is seed images for the three copies (A-C) and the corresponding projections (the Radon transforms) (D). The second row is the reconstruction results of the simple averaging method with SART on the three copies (E-G) and the final averaged reconstruction $(\mathrm{H})$. The third row is the masked Fourier magnitude information of the the three copies (I-K) and the final weighted reconstruction (L). The fourth row is the reconstruction results of the proposed CRM with sparse SART on the three copies from the last iteration (M-O) and the final reconstruction $(\mathrm{P})$

Figure 4 shows the reconstruction results on the Shepp-Logan phantom data set. For the groundtruth, the seed images contain clear copies of the object of interest but with various surroundings (Fig. 4(A)(B)(C)). Since the ranges of tilt angles are limited, the projections (the Radon transforms) can only contain limited information for the object of interest (Fig. 4(D)). The reconstructed structures for the three copies are shown in Fig. 4(E)(F)(G), from which it is clear that the object of interest was blurred due to the missing wedge effect. Fig. 4(H) shows the refined structure gained by the
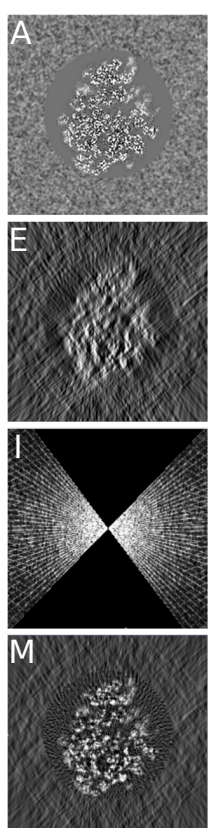
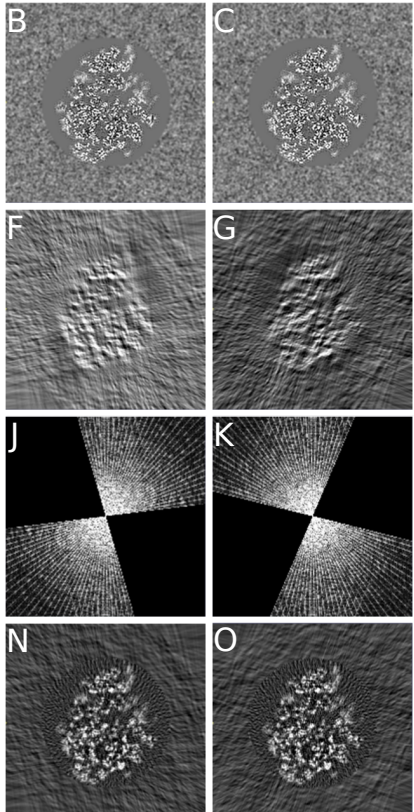
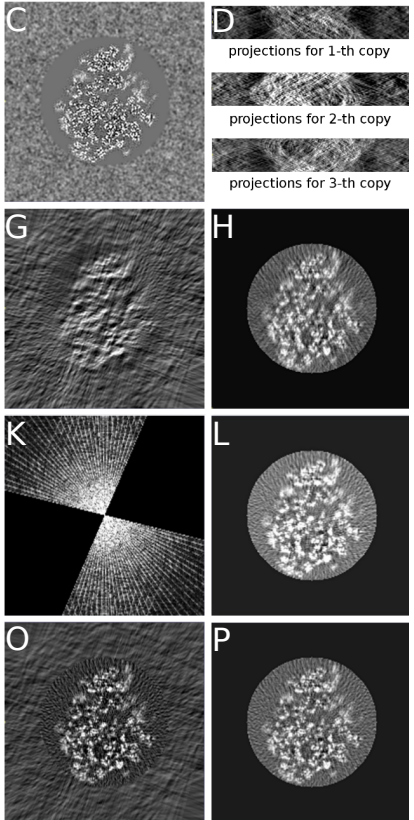

Fig. 5: Comparison of the reconstruction performance on the ribosome structure (EMDB 3489) data set. The first row is seed images for the three copies (A-C) and the corresponding projections (the Radon transforms) (D). The second row is the reconstruction results of the averaging method with SART on the three copies (E-G) and the final averaged reconstruction (H). The third row is the masked Fourier magnitude information of the the three copies (I-K) and the final weighted reconstruction (L). The fourth row is the reconstruction results of the proposed CRM with sparse SART on the three copies from the last iteration (M-O) and the final reconstruction $(\mathrm{P})$ simple averaging method, in which the averaged reconstruction still contains unnatural artifacts and looks very blurry. Then the masked averaging in Fourier is applied to the copies. The masked Fourier magnitude for the three reconstructed structures is shown in Fig. 4(I)(J)(K). Fig. 4(L) shows the refined structure gained by a weighted averaging and inverse transform from these Fourier spectra. Finally, the CRM method is applied to the copies. The sparse SART solution to our CRM started with the first copy and used the reconstructed object of interest from the first copy to initialize the reconstruction of the second copy, which was then used to initialize the reconstruction of the third copy. It then used the reconstruction of the third copy to initialize the first copy for the next iteration and kept doing so, until convergence or the maximal number of iterations was reached (Fig. 4(M)(N)(O)). Fig. 4(P) shows the final refined structure gained by our CRM. By comparing the reconstructed structures of the three methods (Fig. 4(H)(L)(P)), we can find that both the masked averaging in Fourier and our CRM method produce much clearer details than the simple averaging method. However, the result of masked averaging in Fourier still keeps blurry lattice artifacts inside the structure, while the result of CRM presents much smoother structure and looks much closer to the groundtruth phantom.

Figure 5 shows the reconstruction results on the ribosome structure from EMDB 3489. Rather than the block-stained way of adding artifacts, here we added random noise to the seed images (Fig. 5(A)(B)(C)). Similar conclusions can be drawn as in Fig. 4. Both the masked averaging in Fourier and CRM outperforms the simple averaging method (Fig. 5(H)(L)(P)). However, the result of CRM keeps better shape similar to the groundtruth ribosome structure.
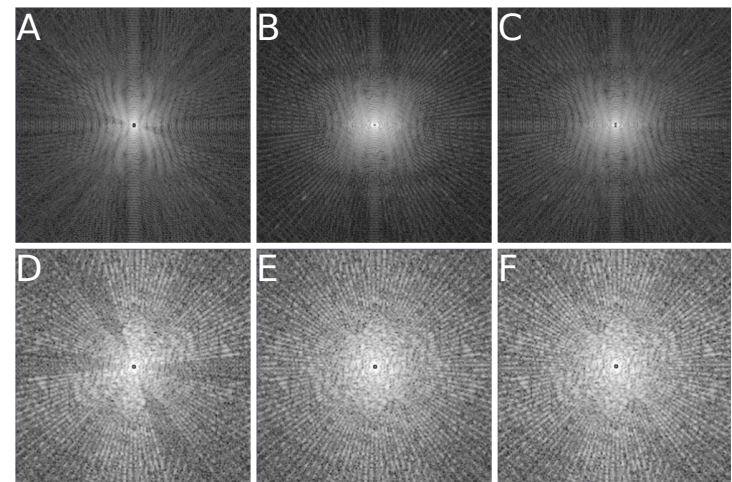

Fig. 6: Comparison of the Fourier transformed (FT) maps of the final reconstructions by the simple averaging (left), masked averaging in Fourier (middle) and CRM (right). The top row are from the results from the Shepp-Logan phantom dataset and the bottom row are the results from the ribosome structure dataset. (A)\&(B)\&(C) are the Fourier transformed maps of the results of simple averaging method (Fig. 4(H)), masked averaging in Fourier (Fig. 4(L)) and CRM (Fig. 4(P)) respectively. (D)\&(E)\&(F) are the Fourier transformed maps of the results of simple averaging method (Fig. 5(H)), masked averaging in Fourier (Fig. 5(L)) and CRM (Fig. 5(P)) respectively.

We further analyzed the results of the averaging method and CRM in the Fourier space. Figure 6 shows the corresponding Fourier transform (FT) maps of the two methods. It can be clearly found that the simple averaging method has a significant number of clearly missing wedges (Fig. 6(A)(D)), whereas the missing wedge effect has been drastically suppressed by the masked averaging in Fourier (Fig. 6(B)(E)) and the CRM method (Fig. $6(D)(F))$. Because the masked averaging in Fourier directly operates on the Fourier weights, it seems that the results of the masked averaging have a more uniform distribution of the signals than the CRM results (Fig. 6(E) v.s. Fig. 6(F)). However, the ones of the CRM have a better signal density (Fig. 6(C) v.s. Fig. 6(B)).

The results of the FT analysis are consistent with the conclusions above, which explain the superior performance of the CRM framework over the simple averaging method. In the following, we would make a further comparison between the CRM framework and the masked averaging in Fourier.

\subsection{Quantitative evaluation}

We evaluated the proposed method by quantitative measures, including the Pearson's inner-product correlation coefficient (PCC), the structural 

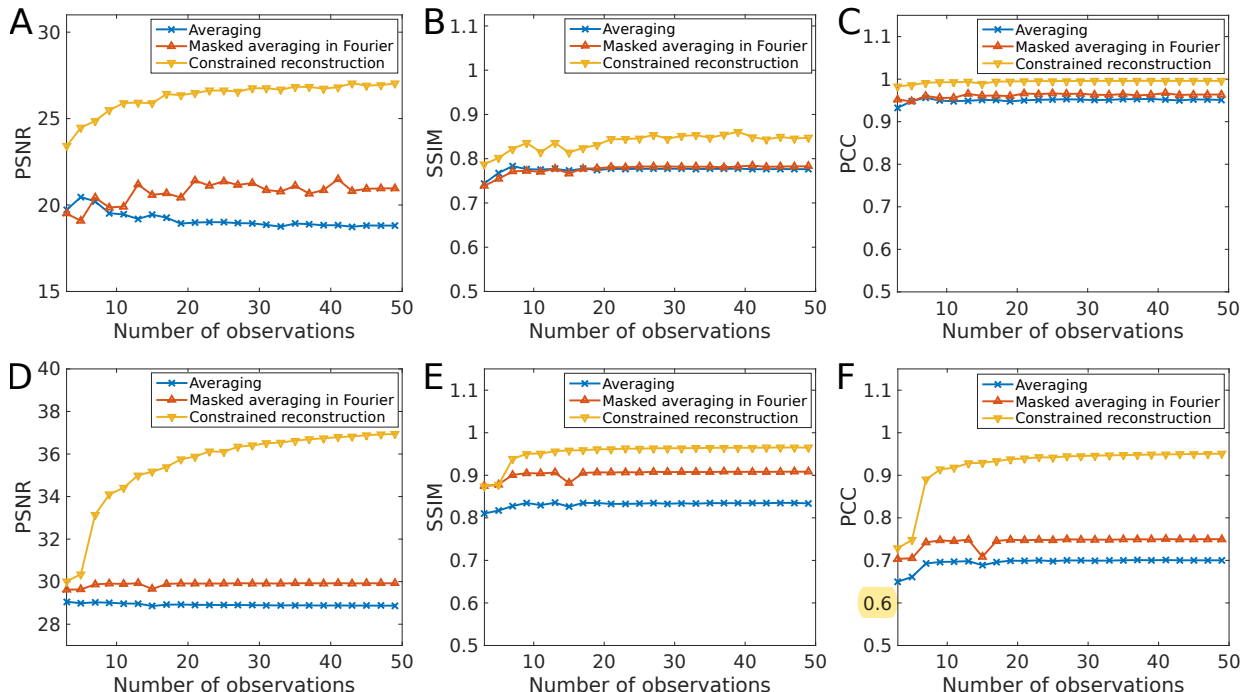

Fig. 7: Parameter sensitivity analysis of the number of copies on the two data sets. Here, the tilt angle range is set to from $-50^{\circ}$ to $+50^{\circ}$ with an interval of $3^{\circ}$. The number of copies ranges from 3 to 49 with a step size of 2. (A)(B)(C): The PSNR, SSIM and PCC curves on the Shepp-Logan phantom data set with respect to the number of copies, respectively. (D)(E)(F): The PSNR, SSIM and PCC curves on the ribosome structure data set with respect to the number of copies, respectively.

similarity (SSIM), and the peak signal-to-noise ratio (PSNR) between the reconstruction and the ground-truth in the seed image.

We measured the reconstructions of the three methods on the two data sets as shown in Fig. 4(H)(L)(P) and Fig. 5(H)(L)(P). Table 1 summarizes the comparison results. The results of the masked averaging in Fourier gain very close SSIM scores to the ones of CRM. However, in general, CRM outperforms the other two methods on both data sets in terms of all the evaluation measures.

\begin{tabular}{c|c|ccc|ccc}
\hline \multicolumn{2}{c|}{ Data set } & \multicolumn{3}{c|}{ Shepp-Logan phantom } & \multicolumn{3}{c}{ Ribosome structure } \\
\hline \multicolumn{2}{c|}{ Measure } & PSNR & SSIM & PCC & PSNR & SSIM & PCC \\
\hline \multirow{3}{*}{ Method } & AVG & 19.72 & 0.74 & 0.93 & 29.06 & 0.81 & 0.65 \\
& M-AVG & 19.72 & 0.74 & 0.95 & 29.62 & $\mathbf{0 . 8 7}$ & 0.70 \\
& CRM & $\mathbf{2 3 . 4 1}$ & $\mathbf{0 . 7 9}$ & $\mathbf{0 . 9 8}$ & $\mathbf{3 0 . 0 2}$ & $\mathbf{0 . 8 7}$ & $\mathbf{0 . 7 3}$ \\
\hline
\end{tabular}

Table 1. Performance comparison between the simple averaging method (AVG), the masked averaging in Fourier (M-AVG) and CRM in terms of PSNR, SSIM and PCC on the two data sets. The best performance is shown in bold.

We further conducted parameter sensitivity analysis to test the robustness of our method with respect to the number of copies, the range of the tilt angles for each copy, and the noise level.

4.2.1 Effect of the number of copies We generated data sets with different numbers of copies (i.e., the subtomograms), ranging from 3 to 49 with a step size of 2 . In this experiment, we fixed the tilt angle range for each copy as $-50^{\circ} \sim+50^{\circ}$ with an interval of $3^{\circ}$. Fig. 7 shows the PSNR, SSIM and PCC curves with the changing number of copies.

It can be seen that the reconstructions produced by CRM always have better performance than that of the simple averaging and the masked averaging in Fourier method regardless of the number of copies. When the number of copies increases from 3 to 15 , the PSNR of CRM quickly improves (Fig. 7(A)(D)). The reason is that at the beginning, a small number of copies is not sufficient to compensate for the lost information due to the missing wedge issue. When the number of copies is more than 15, there is still clear improvement in PSNR of CRM although the speed of the improvement is slower. In contrast, the PSNR of the simple averaging and the masked averaging in Fourier method cannot benefit from the increase in the number of copies, though the masked averaging in Fourier method generally behaves much better than the simple averaging. The stagnation of the quality improvement for the two averaging methods is consistent with previous findings (Bharat et al., 2015) that the quality of the tomograms themselves is a limiting factor for the resolution of the subtomogram averaging. This demonstrates that CRM can effectively integrate information from different copies to compensate for the missing wedge issue whereas the averaging method cannot do so. Similar conclusions can be drawn from the results in terms of SSIM (Fig. 7(B)(E)). Additionally, on the ribosome structure data set, CRM can generate reconstructions with very high structural similarities (close to 1 )

when the number of copies is higher than 20. For PCC, CRM can almost always generate reconstructions that are perfectly correlated $(\mathrm{PCC} \approx 1)$ with the ground-truth regardless of the number of copies. On the ribosome structure data set, the averaging methods stop the quality improvement very early, whereas CRM can still benefit significantly with the increased number of copies (Fig. 7(C)(F)).
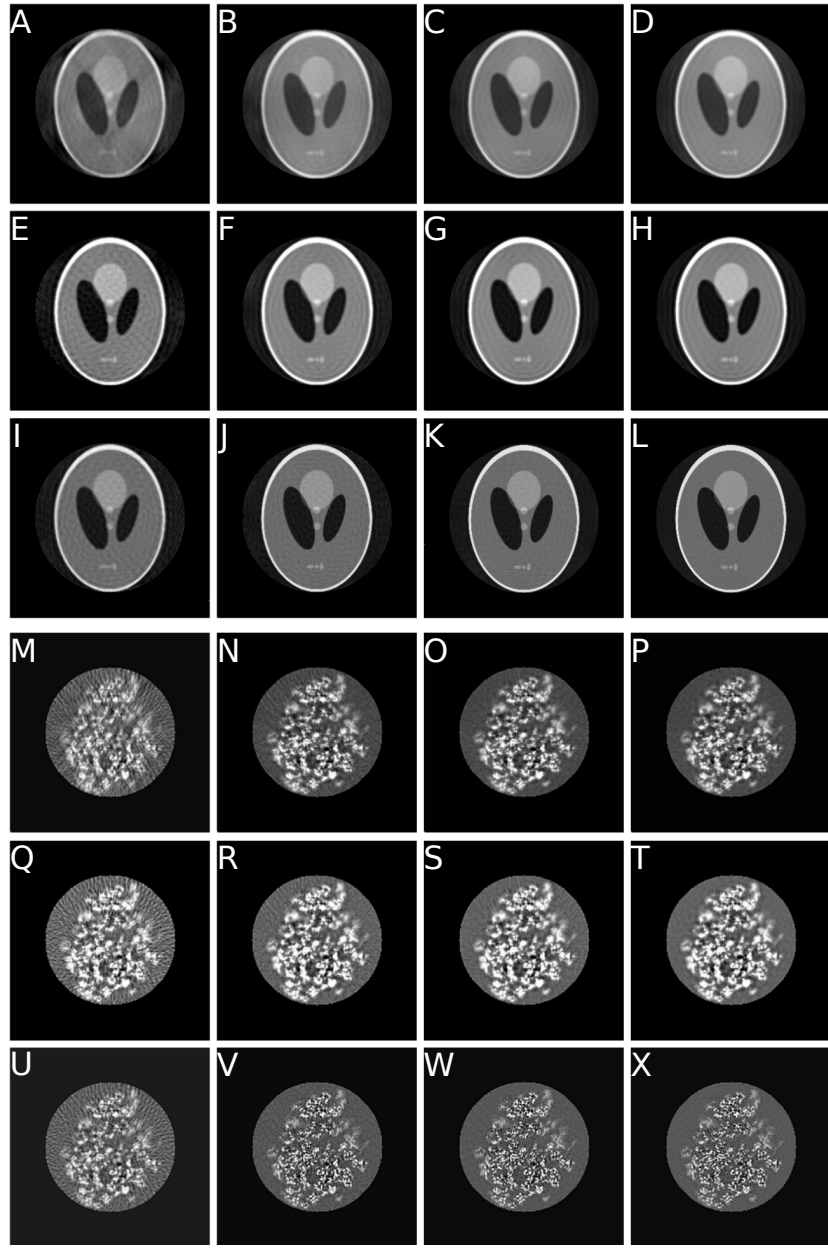
first row is produced by the simple averaging method; the second row is produced by the masked averaging in Fourier; and the third row is produced by CRM. (A)(E)(I)(M)(Q)(U): Reconstruction results with 3 copies. $(B)(F)(J)(N)(R)(V)$ : Reconstruction results with 11 copies. $(C)(G)(K)(O)(S)(W)$ : Reconstruction results with 21 copies. $(D)(H)(L)(P)(T)(X)$ : Reconstruction results with 49 copies. 

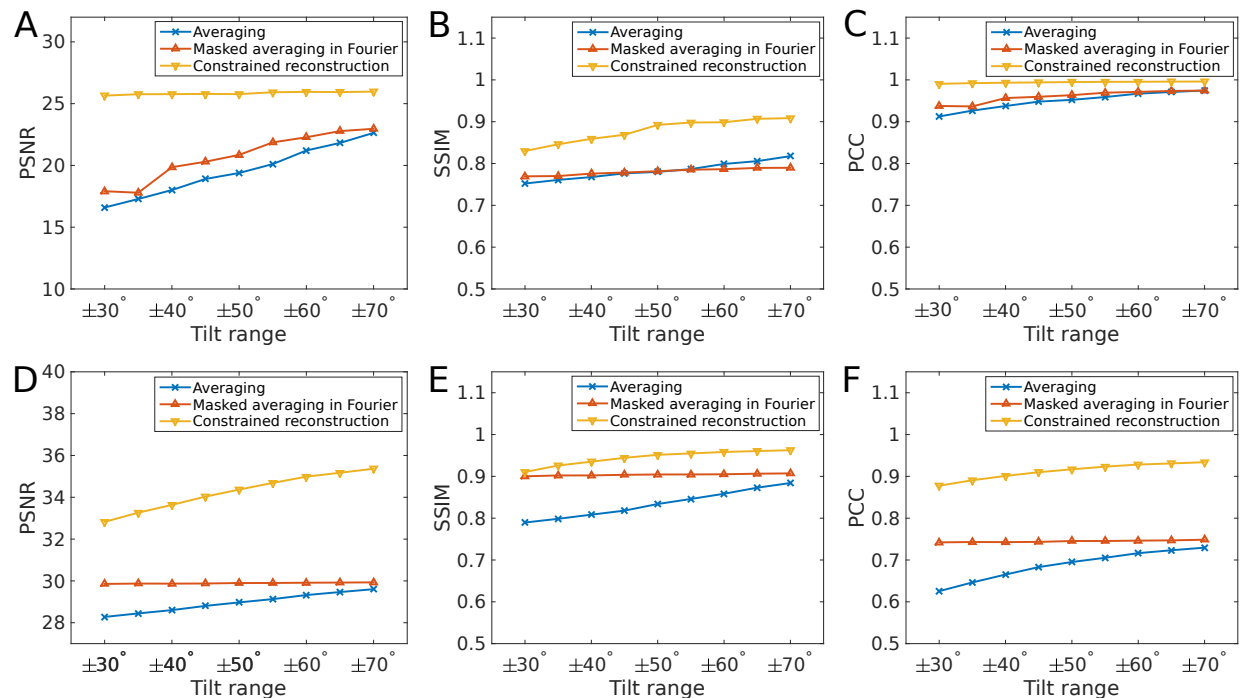

Fig. 9: Parameter sensitivity analysis of the range of the tilt angle on the two data sets. Here, the number of copies is fixed as 11 . The range of the tilt angle changes from $\pm 30^{\circ}$ to $\pm 70^{\circ}$ with a step size of $5^{\circ}$ (for each copy, the interval of tilt angle is fixed to $3^{\circ}$ ). (A)(B)(C): The PSNR, SSIM and PCC curves on the Shepp-Logan phantom data set with respect to the tilt angle range, respectively. (D)(E)(F): The PSNR, SSIM and PCC curves on the ribosome structure data set with respect to the tilt angle range, respectively.
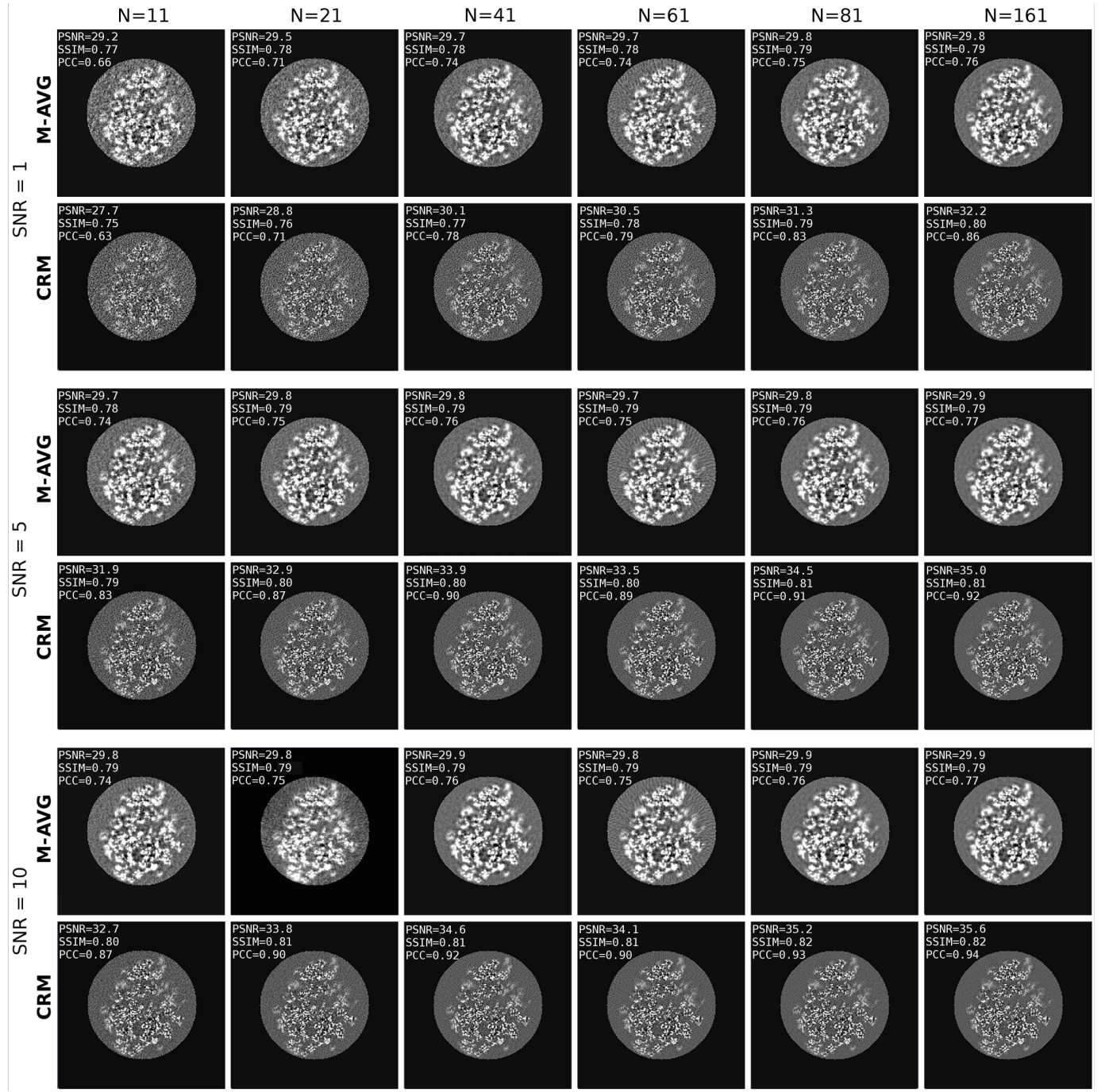

Fig. 10: The performance of reconstructions under various levels of noise and various numbers of copies. AVG stands for the averaging method and CRM stands for the constrained reconstruction model. Both models were solved by SART with 40 iterations and the relaxation parameter of 0.2. PSNR stands for the peak signal-to-noise ratio between the reconstruction and the ground-truth in the seed image; SSIM stands for the structural similarity; and PCC stands for the Pearson's inner-product correlation coefficient.

Fig. 8 shows selected reconstruction results of the three methods with four different numbers of copies, 3, 11, 21 and 49. Consistent with the conclusions from Fig. 7, the reconstructions produced by CRM are much clearer and more similar to the groundtruth compared to the ones produced by the two averaging methods. An interesting observation is that the results produced by the masked averaging in Fourier still carry the bell-ring artifacts (Fig. $8(\mathrm{~F})(\mathrm{G})(\mathrm{H}))$, though the specious information within the missing wedge for each copy has been masked. One hypothesis is that these artifacts come from the stained backgrounds during the iterative optimization of the similarity in reconstruction for each copy, in which the mutual information 
within the whole system is discarded. The averaging methods achieve little visual improvement when the number of copies increases from 21 to 49. In contrast, CRM can continuously improve the quality of the reconstructions when more copies are available.

4.2.2 Effect of the tilt angle range Next, we fixed the number of copies to be 11 and changed the tilt angle range from $\pm 30^{\circ}$ to $\pm 70^{\circ}$ with a step size of $5^{\circ}$ (for each copy, the interval of the tilt angle is fixed to $3^{\circ}$ ). Fig. 9 shows the PSNR, SSIM and PCC curves with the changing range of the tilt angle.

When the range of the tilt angle is larger, the number of projections per copy is higher and thus the missing wedge effect is less severe. Both the averaging methods and CRM use SART within each copy for reconstruction. As expected, all the three methods have better performance with the increasing range of the tilt angle. Consistent with the conclusions from Fig. 7, CRM outperforms the averaging methods in terms of all the measures regardless of the range of the tilt angle. Again, on the Shepp-Logan phantom data set, CRM can almost always generate perfectly correlated reconstructions within any tilt angle range tested here (Fig. 9(C)).

4.2.3 Effect of noise Understanding the effect of noise is critical to investigate the practical usefulness of a tomography reconstruction algorithm. It is related to the information theory and the condition number in linear system analysis (Fried, 1972). Here, we evaluated the effect of noise on our CRM by measuring the performance of CRM under different levels of noise and different numbers of copies.

Due to its abundance in ultra-structures, the ribosome structure data set was chosen in this experiment. For each copy, the projection images were then generated from the stained seed image within the $\pm 50^{\circ}$ tilt angle range with an interval of $3^{\circ}$. And different levels of Gaussian random noise were added to the projection images, with SNR 1, 5 and 10. For a fair comparison, both the averaging method and our CRM used SART with 40 iterations and the relaxation parameter of 0.2. Because the masked averaging in Fourier has been widely used in practice and achieves better results than the simple averaging method, here we mainly present the comparison between the $\mathrm{CRM}$ and the masked averaging in Fourier.

Fig. 10 shows the reconstruction results of the two methods under different levels of noise and with different numbers of copies. In terms of PSNR and PCC, CRM almost always outperforms the averaging method, except for the high noise but with a small number of copies $(S N R=1$ and $N \leq 61$ ). This implies that when the noise level is very high, a very small number of copies is not sufficient for CRM to compensate for the loss in the reconstruction resolution caused by the noise. However, if a sufficient number of copies is given, CRM can effectively utilize the information in different copies regardless of the level of noise. Given a certain noise level, the performance of CRM increases quickly with the increasing number of copies, whereas the masked averaging method cannot benefit from such additional information. This phenomenon has also been observed in our previous experiments (Fig. 8 and Fig. 9), which may need further efforts to explain. In terms of SSIM, both methods have comparable values. These results demonstrate that while both the averaging method and CRM can well recover the main structure of the subtomogram, CRM is robust to the noise and can take great advantage of the multiple copies to reach a much higher quality of reconstruction than the averaging method.

4.2.4 Convergence analysis Except for the reconstruction accuracy, the convergence and run speed is also an important issue for a novel iterative method. Because the solution of the constrained reconstruction framework inherits the features from the algebraic reconstruction technique, its convergence depends on the internal update strategy. Considering the usage of SART in the internal loop of CRM and the exact proof of SART's convergence (Jiang and Wang, 2003), the sparse SART solution to CRM (CRM-SART) always has a converged station.

Theoretically, CRM-SART will have similar convergence rate with the original SART algorithm. Here, we chose the CRM reconstruction of 49 copies from each dataset with the tilt angle range from $-50^{\circ}$ to $50^{\circ}\left(3^{\circ}\right.$ interval) as examples and use the simple separate reconstruction of each copy as the comparison. The iterative number from 1 to 80 are tested and the root mean square (RMS) of the reprojection error in each turn of iteration
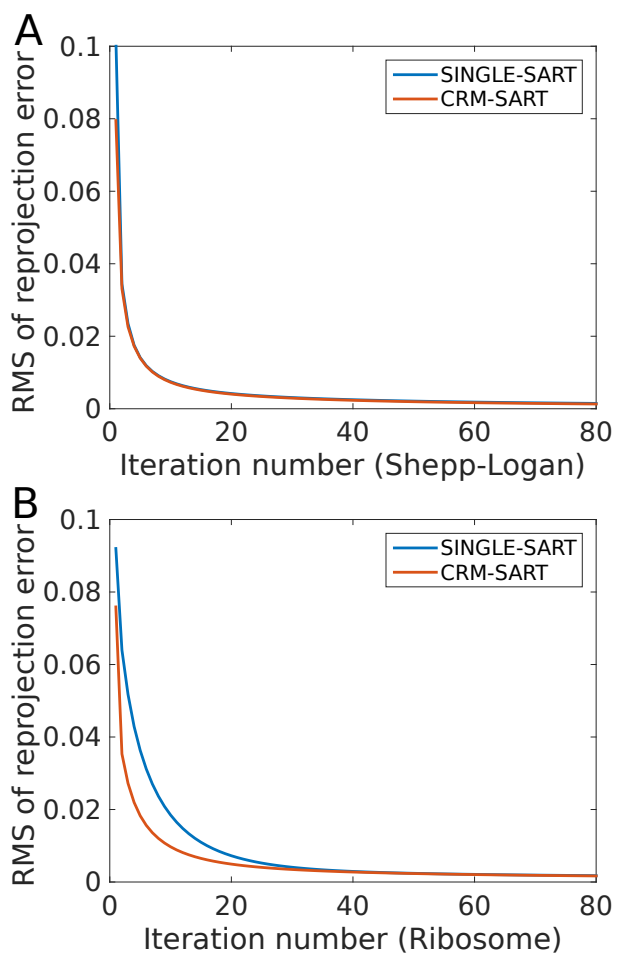

Fig. 11: The comparison of reprojection error under different iteration number. (A) Results from the dataset of Shepp-Logan phantom; (B) Results from the dataset of ribosome structure.

is calculated. the RMS of the reprojection error is calculated as follows

$$
\varepsilon=\left[\frac{1}{M N} \sum_{i=1}^{M} \sum_{j=1}^{N}\left(v_{i j}-v_{i j}^{\prime}\right)^{2}\right]^{\frac{1}{2}}
$$

where $v_{i j}$ is the $i$ th pixel in the $j$ th experimental projection image, $v_{i j}^{\prime}$ is the corresponding calculated projection, $M$ is the number of pixels in each projection and $N$ is the number of projections. The value change of the reprojection error for each dataset is shown in Figure 11. It can be found that for each dataset, the CRM-SART and the original SART always reach the convergence after about 40 iterations. Especially, the CRM-SART almost finishes the convergence within about 20 iterations. In the dataset of Ribosome, CRM-SART gains faster convergence rate than the original SART, which may be caused by the complex structure inside the ribosome dataset and the exchange of initial model within the CRM framework. It should be noted that the CRM always produce smaller reprojection error than the original SART. By the experiment, it can be concluded that an iteration number within $20 \sim 40$ is enough for convergence for the SART solution of CRM. For the relaxation parameter, a general value of 0.2 (as that in the original SART) is almost enough for CRM-SART.

\subsection{D case study}

We have proved the robustness of our constrained reconstruction model under different conditions. Here, we further extend the constrained reconstruction model to three-dimensional cases and try to analyze its performance. The performance of CRM is compared with that of masked averaging in Fourier. To avoid the effect of the non-structural sharp cutting edge of an EMDB density map in the estimation of resolution, we use the PDB-converted structures instead of EMDB maps in our following experiment, which are produced by EMAN2's e2pdb2mrc.py (Tang et al., 2007).

Two different structures are used, including the MscS channel structure of Escherichia coli converted from PDB 5Y4O (Yu et al., 2018) and the GTP-binding nuclear protein Ran (protein) converted from PDB 5ZPU. A modified InSilicoTEM (Vulović et al., 2013) is used to simulate the tilt series, in which the volumes of particles (subtomograms) are stained by noise and artificial membranes. To focus on the effects of the reconstruction model, the effect of the contrast transfer function (CTF) is prefixed. For each structure, volumes with 200 particles under different positions are simulated. 

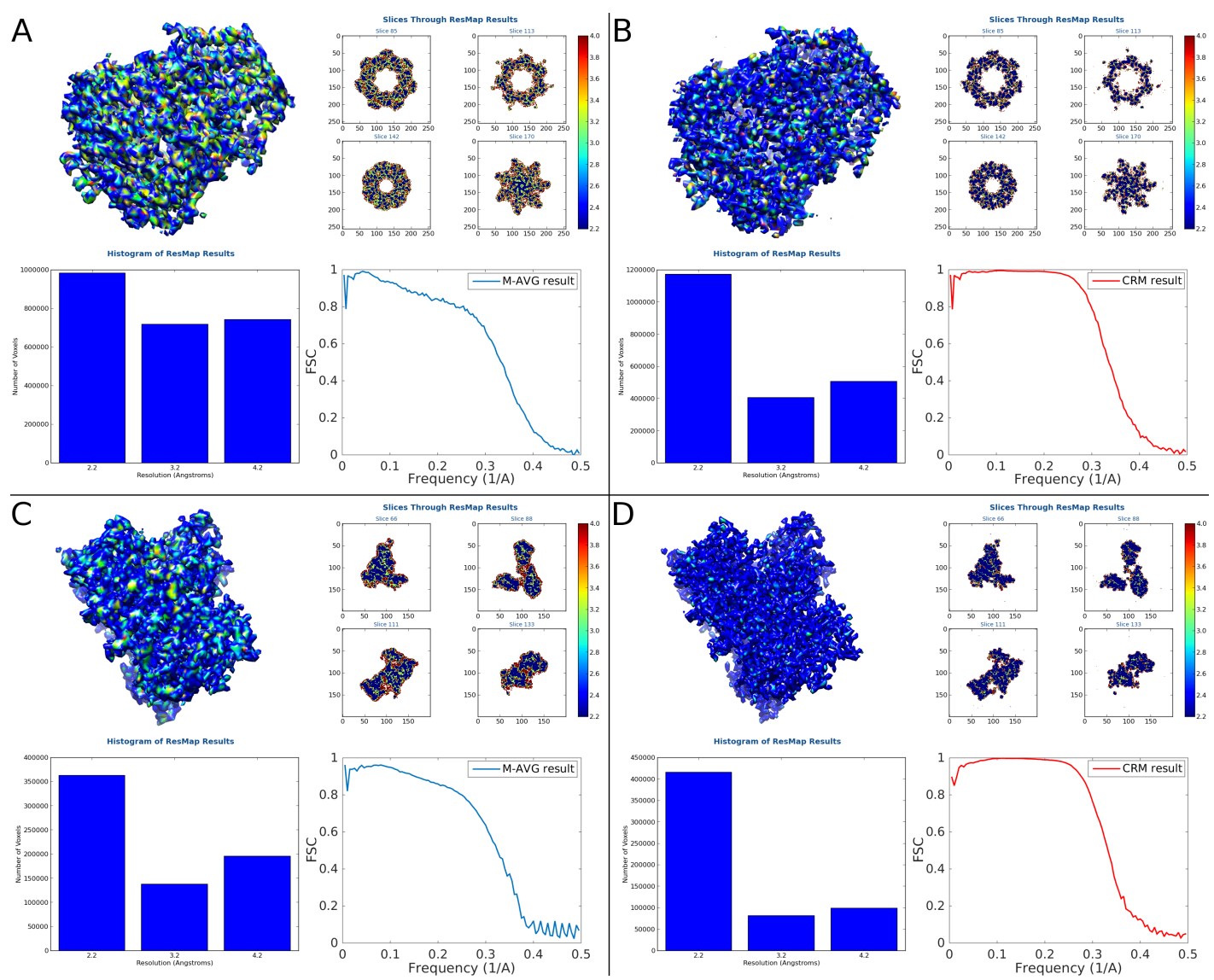

Fig. 12: The 3D reconstruction results and their detailed analysis of FSC and ResMap. (A)\&(B) are the reconstruction results of the MscS channel structure converted from PDB 5Y4O. (C)\&(D) are the reconstruction results of the GTP-binding nuclear protein Ran converted from PDB 5ZPU. Here, (A)\&(C) are results carried out by the masked average in Fourier whereas (B)\&(D) are results carried out by our constrained reconstruction model.

The signal to noise ratio of the projections is controlled around $1: 1$ and the tilt range is set to $-50^{\circ}+50^{\circ}$ with $5^{\circ}$ intervals.

Since the geometry position for each subvolume and the projection parameters are known in prior during simulation, it is not difficult to obtain an averaged subtomogram by masked averaging in Fourier for the particles as well as the accurate projection parameters for each particle in our CRM. To analyze the results, both Fourier Shell Correlation (FSC) and ResMap (Kucukelbir et al., 2014) were used to compare the performance between the masked averaging in Fourier and our CRM. For each reconstruction result, a sphere mask was applied to suppress the effects of unnecessary surroundings, and the ground-truth that converted from the PDB was used as the reference. All the results were calculated with the $\AA$ /voxel setting to 1 .

Figure 12 shows the reconstruction results for both the methods. For a fair comparison, both the averaging method and our CRM used SART with 40 iterations and the relaxation parameter of 0.2 . Judging from the 3D structures shown in Figure 12, we can find that the masked averaging in Fourier and our CRM achieved similar structures. However, the results of CRM has a clearer boundary and detailed structures. The ResMap results and FSC curves show that the average method didn't achieve as high resolution as our CRM for both the MscS channel structure and the GTP-binding nuclear protein Ran. According to the frequency value truncated at $0.5\left(\mathrm{FSC}_{0.5}\right)$, for the MscS channel structure, the result of the masked averaging in Fourier has a resolution of $3.08 \AA$, while the result reconstructed by our CRM has a resolution of $3.0 \AA$; for the GTPbinding nuclear protein, the result of the masked averaging in Fourier has a resolution of $3.05 \AA$, while the result reconstructed by our CRM has a resolution of $2.98 \AA$. Furthermore, it can be found that the FSC curves generated by CRM generally have higher correlation values compared with the ones of the averaging method, which indicates that the results of CRM are much closer to the groundtruth. In details, the local resolution distributions generated by ResMap show that the results of CRM have the local resolutions mainly distributed at $2.2 \AA$ and $3.2 \AA$, while the results of the averaging method have a lot of local resolutions distributed at $4.2 \AA$. Moreover, the ResMap reported a mean resolution of $3.10 \AA$ and $2.96 \AA$ for the masked averaging in Fourier's results of the MscS channel structure and the GTP-binding nuclear protein, respectively. As a comparison, the reported mean resolutions of our CRM are $2.96 \AA$ and 2.77 $\AA$ for the results of the MscS channel structure and the GTP-binding nuclear protein, respectively. Here, both the results of FSC and ResMap indicate that our constrained reconstruction model can gain an improvement in the reconstruction quality compared to the masked averaging in Fourier.

\section{CONCLUSION}

In this paper, we proposed a novel constrained reconstruction model for high-resolution subtomogram averaging. Our model can alleviate the missing wedge issue caused by the limited tilt angle range by effectively integrating information from multiple copies of the object of interest. We gave the first theoretical proof to guarantee that the solution space of our model is no larger, and in practice much smaller, than that of the averaging method. We then proposed a sparse Kaczmarz algorithm-based solution for our model and extended it to a novel sparse SART solution, which is used in our final framework. It should be noted that although our solution can compensate for the missing wedge effect, it does not use any prior knowledge. Our solution only focuses on the reinforcement of natural information underlying the large number of projections. Therefore, our proposal will not introduce any false information related to the presumption of prior knowledge. Experimental results demonstrate the effectiveness, robustness and generalization power of our model, which promise its huge potential for high-resolution subtomogram averaging.

The proposed constrained reconstruction model is focused on the final information fusion in reconstruction. Here, we don't try to change the optimization of ultrastructure's orientation and geometry in the alignment procedure. Instead, we could use the classic averaging in the parameter refinement but use the CRM in the final information fusion. We try to deliver the message that the general reconstructing and averaging maybe not 
enough. Especially, the existence of ring-bell artifacts and the stagnation of the quality improvement in the averaging method may require further comprehensive study. Furthermore, though we have proposed the concept of constrained reconstruction, we do not investigate any prior information and regulation term (for example, L1-form for sparsity and nonuniform sampling) within the model. Further research to better understand the underlying mechanism of constrained reconstruction is needed, as well as the combination with other optimization techniques. Considering the complex situation in cryo-electron microscopy, further efforts are required to apply the constrained reconstruction into practical application.

\section{REFERENCES}

Andersen, A. and Kak, A. (1984). Simultaneous algebraic reconstruction technique (sart): A superior implementation of the art algorithm. Ultrasonic Imaging, 6(1), 81 -94 .

Andersen, A. H. (1989). Algebraic reconstruction in ct from limited views. IEEE transactions on medical imaging, 8(1), 50-55.

Bartesaghi, A., Lecumberry, F., Sapiro, G., and Subramaniam, S. (2012). Protein secondary structure determination by constrained single-particle cryo-electron tomography. Structure, 20(12), 2003-2013.

Beister, M., Kolditz, D., and Kalender, W. A. (2012). Iterative reconstruction methods in X-ray ct. Physica medica, 28(2), 94-108.

Bharat, T. A. and Scheres, S. H. (2016). Resolving macromolecular structures from electron cryo-tomography data using subtomogram averaging in relion. Nat. Protoc., 11(11), 2054

Bharat, T. A., Russo, C. J., Löwe, J., Passmore, L. A., and Scheres, S. H. (2015) Advances in single-particle electron cryomicroscopy structure determination applied to sub-tomogram averaging. Structure, 23(9), 1743-1753.

Briggs, J. A. (2013). Structural biology in situ: the potential of subtomogram averaging. Curr. Opin. Struc. Biol., 23(2), $261-267$.

Carazo, J.-M. (1992). The fidelity of $3 \mathrm{~d}$ reconstructions from incomplete data and the use of restoration methods. In Electron tomography, pages 117-164.

Chen, Y. and Forster, F. (2014). Iterative reconstruction of cryo-electron tomograms using nonuniform fast fourier transforms. J. Struct. Biol., 185(3), 309 - 316.

De Rosier, D. J. and Klug, A. (1968). Reconstruction of three dimensional structures from electron micrographs. Nature, 217(1), 130-134.

Deng, Y., Chen, Y., Zhang, Y., Wang, S., Zhang, F., and Sun, F. (2016). Icon: $3 \mathrm{~d}$ reconstruction with missing-information restoration in biological electron tomography. J. Struct. Biol., 195(1), $100-112$.

Förster, F., Medalia, O., Zauberman, N., Baumeister, W., and Fass, D. (2005). Retrovirus envelope protein complex structure in situ studied by cryo-electron tomography. Proceedings of the National Academy of Sciences, 102(13), 47294734.

Frank, J. (2006). Electron tomography: methods for three-dimensional visualization of structures in the cell. Springer.

Fried, I. (1972). Condition of finite element matrices generated from nonuniform meshes. AIAA J., 10(2), 219-221.

Galaz-Montoya, J. G. and Ludtke, S. J. (2017). The advent of structural biology in situ by single particle cryo-electron tomography. Biophysics Reports, 3(1), 17-35.

Gilbert, P. (1972). Iterative methods for the three-dimensional reconstruction of an object from projections. J. Theor. Biol., 36(1), $105-117$.

Gordon, R., Bender, R., and Herman, G. T. (1970). Algebraic reconstruction techniques (art) for three-dimensional electron microscopy and x-ray photography J. Theor. Biol., 29(3), $471-481$.

Han, R., Wan, X., Wang, Z., Hao, Y., Chen, Y., Gao, X., Liu, Z., Ren, F., Sun, F., and Zhang, F. (2017). Autom: a novel automatic platform for electron tomography reconstruction. J. Struct. Biol., 199(3), 196-208.

Harris, W., Zhang, Y., Yin, F.-F., and Ren, L. (2017). Estimating 4d-cbct from prior information and extremely limited angle projections using structural pca and weighted free-form deformation for lung radiotherapy. Medical Physics, 44(3), 1089-1104.

James, N. R., Brown, A., Gordiyenko, Y., and Ramakrishnan, V. (2016). Translational termination without a stop codon. Science, 354(6318), 1437-1440.

Jiang, M. and Wang, G. (2003). Convergence of the simultaneous algebraic reconstruction technique (sart). IEEE T. Image Process., 12(8), 957-961.

Kaczmarz, S. (1937). Angenäherte auflösung von systemen linearer gleichungen. Bulletin International de l'Académie Polonaise des Sciences et des Lettres, 35 , 355-357.

Kovacik, L., Kerieche, S., Hoog, J. L., Juda, P., Matula, P., and Raska, I. (2014). A simple fourier filter for suppression of the missing wedge ray artefacts in single-axis electron tomographic reconstructions. J. Struct. Biol., 186(1), $141-152$.

Kucukelbir, A., Sigworth, F. J., and Tagare, H. D. (2014). Quantifying the local resolution of cryo-em density maps. Nature methods, 11(1), 63.

Kunz, M. and Frangakis, A. S. (2014). Super-sampling sart with ordered subsets. J. Struct. Biol., 188(2), 107-115.

Kupsch, A., Lange, A., Hentschel, M. P., Lück, S., Schmidt, V., Grothausmann, R., Hilger, A., and Manke, I. (2016). Missing wedge computed tomography by iterative algorithm directt. J. Micro., 261(1), 36-45.

Leary, R., Saghi, Z., Midgley, P. A., and Holland, D. J. (2013). Compressed sensing electron tomography. Ultramicroscopy, 131, $70-91$.

Penczek, P. A., Renka, R., and Schomberg, H. (2004). Gridding-based direct fourier inversion of the three-dimensional ray transform. JOSA A, 21(4), 499-509.

Prakash, P., Kalra, M. K., Kambadakone, A. K., Pien, H., Hsieh, J., Blake, M. A., and Sahani, D. V. (2010). Reducing abdominal ct radiation dose with adaptive statistical iterative reconstruction technique. Investigative radiology, 45(4), 202-210.

Scheres, S. H. (2012). Relion: implementation of a bayesian approach to cryo-em structure determination. J. Struct. Biol., 180(3), 519-530.

Schmid, M. F. and Booth, C. R. (2008). Methods for aligning and for averaging 3d volumes with missing data. Journal of structural biology, 161(3), 243-248.

Shepp, L. A. and Logan, B. F. (1974). The fourier reconstruction of a head section. IEEE T. Nucl. Sci., 21(3), 21-43.

Shimizu, K., Mulkern, R. V., Oshio, K., Panych, L. P., Yoo, S.-S., Jolesz, F. A., and Kikinis, R. (1998). Rapid tip tracking with mri by a limited projection reconstruction technique. J. Magn. Reson. Imaging, 8(1), 262-264.

Tang, G., Peng, L., Baldwin, P. R., Mann, D. S., Jiang, W., Rees, I., and Ludtke, S. J. (2007). Eman2: an extensible image processing suite for electron microscopy. $J$. Struct. Biol., 157(1), 38-46.

Vulović, M., Ravelli, R. B., van Vliet, L. J., Koster, A. J., Lazić, I., Lücken, U., Rullgård, H., Öktem, O., and Rieger, B. (2013). Image formation modeling in cryo-electron microscopy. J. Struct. Biol., 183(1), 19-32.

Wu, T., Stewart, A., Stanton, M., McCauley, T., Phillips, W., Kopans, D. B., et al. (2003). Tomographic mammography using a limited number of low-dose conebeam projection images. Medical Physics, 30(3), 365-380.

$\mathrm{Xu}, \mathrm{M}$. and Alber, F. (2012). High precision alignment of cryo-electron subtomograms through gradient-based parallel optimization. BMC systems biology, 6(1), S18.

Xu, M., Beck, M., and Alber, F. (2012). High-throughput subtomogram alignment and classification by fourier space constrained fast volumetric matching. J. Struct. Biol., 178(2), 152-164.

Yu, J., Zhang, B., Zhang, Y., Xu, C.-q., Zhuo, W., Ge, J., Li, J., Gao, N., Li, Y., and Yang, M. (2018). A binding-block ion selective mechanism revealed by a na/k selective channel. Protein $\mathcal{E}$ cell, 9(7), 629-639.

Zhu, N., Jiang, Y., and Kato, S. (2005). Ultrasonic computerized tomography (ct) for temperature measurements with limited projection data based on extrapolated filtered back projection (fbp) method. Energy, 30(2), 509 - 522. 